\title{
Solvent Extraction Chemistry of Dialkyldithiophosphates. II. Evaluation of Two-phase Titration Data for Dibutyl- and Methylheptyldithiophosphoric Acids According to the Solubility Parameter Concept
}

\author{
STIG WINGEFORS
}

Department of Nuclear Chemistry, Chalmers University of Technology, Fack, S-41296 Göteborg 5, Sweden

The general principles for the evaluation of solubility parameters $(\delta)$ of acidic extraction reagents from two-phase titrations are discussed. When the method of this work is applied to data for dibutyl- and methylheptyldithiophosphoric acids $\delta=20.7 \mathrm{~J}^{1 / 2}$ $\mathrm{cm}^{-3 / 2}$ is obtained for both isomers.

The partition of chelating metal extractants plays an important part in the analysis of metal complexation, but also in the analysis of metal complex behaviour in the organic phase. In a previous paper ${ }^{1}$ it was shown how the "apparent $\mathrm{p} k_{\mathrm{a}}$ " or $\log k_{\mathrm{d}} k_{\mathrm{a}}^{-1}$ (where $k_{\mathrm{d}}$ and $k_{\mathrm{a}}$ are the partition and dissociation constants of the reagent, HA) may be obtained with high accuracy from two-phase titrations. $^{2}$ The aim of this paper is to show how the two-phase titration data may be interpreted according to the solubility parameter concept. Similar studies were originally performed by Suzuki and co-workers, ${ }^{3,4}$ who used separately determined $k_{\mathrm{d}}$ values of a series of $\beta$-diketones for this purpose. Two-phase titration data were used by SkytteJensen in a study of 1-phenyl-3-methyl-4-acyl-5pyrazolones. ${ }^{5}$ However, both of them employed reagents capable of keto-enol tautomerization, which to some extent obscured the treatment of data and probably also influenced the final conclusions. This problem does not exist for the dialkyldithiophosphoric acids. Furthermore, some of the earlier assumptions, especially concerning the handling of aqueous phase interactions, need reexamination.
The determination of $\log k_{\mathrm{d}} k_{\mathrm{a}}^{-1}$ for dibutyldithiophosphoric acid (HDBDTP) and methylheptyldithiophosphoric acid (HMHpDTP) has been described in the first paper of this series ${ }^{1}$ the values needed for the actual purpose are given in the second column of Table 1 .

The Scatchard-Hildebrand (SH) equation ${ }^{6-9}$ for regular liquid mixtures reads as eqn. (1), where $\bar{\gamma}_{1}=$ activity coefficient of component (1) in the mol $R T \ln \gamma_{1}=v_{1}\left(\delta_{1}-\delta_{2}\right)^{2} \phi_{2}^{2}$

fraction scale; $v_{1}=$ molal volume of component (1), $\mathrm{cm}^{3} ; \delta_{\mathrm{i}}=$ solubility parameter of (i), $\mathrm{J}^{1 / 2} \mathrm{~cm}^{-3 / 2}$; $\phi_{2}=$ volume fraction of component (2).

In order not to complicate the following discussion too much by transformation of all thermodynamic quantities from the molar to the mol fraction scale, a short-cut procedure simply referring to $k_{\mathrm{d}}$ will be used.

It is easily shown that at small concentrations (negligible on the volume fraction scale) the relationship between the partition constant in the mol fraction scale $\left(=k_{\mathrm{dx}}\right)$ and in the molar scale $\left(=k_{\mathrm{d}}\right)$

$k_{\mathrm{dx}}=k_{\mathrm{d}}\left(v_{\mathrm{s}} / v_{\mathrm{aq}}^{\prime}\right)$

where $v_{\mathrm{S}}$ is the molal volume of organic solvent and $v_{\mathrm{aq}}^{\prime}$ is a constant depending on the composition of the aqueous phase. Actually this quantity need not be known to attempt using the SH-equation. Further, when the pure liquid solute is taken as standard state for both phases, its partition constant is given by eqn. (3), irrespective of 
concentration. (The bar denotes the organic phase.) For infinite dilution the combination of eqns. (1), (2) and (3) leads to eqn. (4).

$k_{\mathrm{dx}}=\gamma / \bar{\gamma}$

$R T \ln \left(k_{\mathrm{d}} \frac{v_{\mathrm{S}}}{v_{\mathrm{aq}}^{\prime}}\right)=R T \ln { }^{\infty} \gamma-v_{\mathrm{HA}}\left(\delta_{\mathrm{HA}}-\delta_{\mathrm{S}}\right)^{2}$

It then follows that

$$
R T \ln \left(k_{\mathrm{d}} k_{\mathrm{a}}^{-1} v_{\mathrm{S}}\right)=A-v_{\mathrm{HA}}\left(\delta_{\mathrm{HA}}-\delta_{\mathrm{S}}\right)^{2}
$$

where $A$ is a constant depending only on aqueous phase parameters: $A=R T \ln \left({ }^{\infty} \gamma \cdot k_{\mathrm{a}}^{-1} v_{\mathrm{aq}}^{\prime}\right)$.

When $v_{\text {HA }}$ is known anyhow, it is certainly not advisable to try a direct fit of eqn. (5) to $k_{\mathrm{d}} k_{\mathrm{a}}^{-1}$ data. When this is done, the determined values of $\delta_{\mathrm{HA}}$ and $v_{\mathrm{HA}}$ might give a good overall fit, but they may be of little physical significance. Also this problem seems to be more or less serious, depending on the set of solvents used. Instead eqn. (5) can be rearranged:

$\frac{R T \ln \left(k_{\mathrm{d}} k_{\mathrm{a}}^{-1} v_{\mathrm{S}}\right)}{v_{\mathrm{HA}}}+\delta_{\mathrm{S}}^{2}=\left[\frac{A}{v_{\mathrm{HA}}}-\delta_{\mathrm{HA}}^{2}\right]+2 \delta_{\mathrm{HA}} \delta_{\mathrm{S}}$

When the left hand side of eqn. (6) is plotted against $\delta_{\mathrm{S}}$, a straight line with slope $=2 \delta_{\mathrm{HA}}$ and the intercept given by the expression within brackets should be found, $c f$. Fig. 1 . The data used are given in Table 1. Inspection of Fig. 1 shows a fair agreement with the assumptions behind the SH equation. However, the mere correlation to a straight line is not conclusive in a case like this. In fact, it might be shown that a similar plotting of data using the geometric mean of $k_{\mathrm{d}} k_{\mathrm{a}}^{-1}$ for all solvents will also give a straight line with only a slightly less good fit! This is of course not peculiar to just the actual experimental data, but rather emphasizes the fact

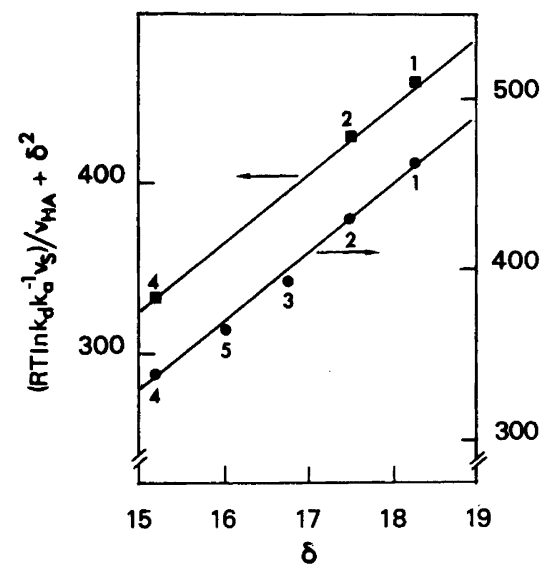

Fig. 1. Plots of the left hand side of eqn. (6) $v s . \delta_{\mathrm{S}}$ for HDBDTP (•) and HMHpDTP (ש). Organic solvents are denoted according to Table 1 . Note the shift of ordinate scales.

that one must be very careful in the application of the SH equation. However, the $\delta_{\mathrm{HA}}$ value obtained from such a plot is too low $(\approx 16)$ and using the experimental data gives $\delta_{\mathrm{HA}} \approx 20$. The $\mathrm{SH}$ equation may, therefore, be considered to give a significant description of the systems.

Cyclohexane and dodecane do not fit in with the other solvents. For dodecane it might be expected that its large volume and long-chained structure will give rise to a contribution in excess entropy not taken into account by regular solution theory and the SH equation. Such phenomena have commonly been thought ${ }^{6,10,11}$ to be described an additional Flory-Huggins term ${ }^{8,9}$ in eqn. (1),

$$
\begin{aligned}
& R T \ln \gamma_{1}=v_{1}\left(\delta_{1}-\delta_{2}\right)^{2} \phi_{2}^{2}+ \\
& R T\left\{\ln \frac{\phi_{1}}{x_{1}}+\phi_{2}\left(1-\frac{v_{1}}{v_{2}}\right)\right\}
\end{aligned}
$$

Table 1. Data needed for the evaluation of eqns. (6) and (8). T $=298 \mathrm{~K} \cdot v_{\mathrm{HA}}=226 \mathrm{~cm}^{3} \cdot{ }^{17}$ The $\log k_{\mathrm{d}} k_{\mathrm{a}}^{-1}$ values refer to $1 \mathrm{M} \mathrm{NaClO}_{4}$ as aqueous phase. ${ }^{1}$

\begin{tabular}{llllll}
\hline Organic solvent & $\begin{array}{l}\log k_{\mathrm{d}} k_{\mathrm{a}}^{-1} \\
\text { HDBDTP }\end{array}$ & HMHpDTP & $\begin{array}{l}\delta_{\mathrm{S}} \\
\mathbf{J}^{1 / 2} \mathrm{~cm}^{-3 / 2}\end{array}$ & $\begin{array}{l}v_{\mathrm{S}} \\
\mathbf{c m}^{3}\end{array}$ & Ref. \\
\hline 1. Toluene & 3.12 & 3.05 & 18.27 & 106.8 & 14 \\
2. Carbontetrachloride & 2.98 & 2.98 & 17.49 & 97.1 & 15 \\
3. Cyclohexane & 2.40 & - & 16.78 & 108.8 & 14 \\
4. Heptane & 2.10 & 1.97 & 15.20 & 147.4 & 16,15 \\
5. Dodecane & 1.94 & - & 16.04 & 226.8 & 16,15 \\
\hline
\end{tabular}


where $x_{1}$ is the mol fraction of (1). This approach has been criticized ${ }^{12}$ and Hildebrand ${ }^{13}$ has also shown it to be of no significance unless long-chained molecules are actually involved. Thus, for dodecane it could be relevant. When eqn. (7) is incorporated into the model, eqn. (6) is replaced by

$$
\begin{aligned}
& \frac{R T\left\{\ln \left(k_{\mathrm{d}} k_{\mathrm{a}}^{-1} v_{\mathrm{HA}}\right)+1-v_{\mathrm{HA}} / v_{\mathrm{S}}\right\}}{v_{\mathrm{HA}}}+ \\
& \delta_{\mathrm{S}}^{2}=\left[\frac{A}{v_{\mathrm{HA}}}-\delta_{\mathrm{HA}}^{2}\right]+2 \delta_{\mathrm{HA}} \delta_{\mathrm{S}}
\end{aligned}
$$

If eqn. (8) is used instead of eqn. (6), the fitting for dodecane is significantly improved but the data for other solvents do not fit as well. Cyclohexane is still the worst example, but it is well-known that this solvent almost invariably behaves as if it has a solubility parameter of $0.2-0.4$ units under the thermodynamic value. The exact reason for this discrepancy is not known, but certainly it has to do with liquid structure more than energetic molecular interactions. Conclusively the data for cyclohexane were excluded from the subsequent linear regression analysis, which gave $\delta_{\mathrm{HA}}=20.8 \pm 0.8$ and $20.1 \pm 0.6$ $\mathrm{J}^{1 / 2} \mathrm{~cm}^{-3 / 2}$ for eqns. (6) and (8), respectively, in the case of HDBDTP. The corresponding values for HMHpDTP are $20.6 \pm 0.2$ and $20.0 \pm 0.5 \mathrm{~J}^{1 / 2} \mathrm{~cm}^{-3 / 2}$. There is clearly no significant difference between the two isomers and pooling all data together gives $20.7 \pm 0.4$ or $20.0 \pm 0.3 \mathrm{~J}^{1 / 2} \mathrm{~cm}^{-3 / 2}$. These values may be taken as the final result for both isomers, and probably also for all straight-chained $\mathrm{C}_{8}$-dialkyldithiophosphoric acids. It is somewhat disturbing that eqns. (6) and (8) give different estimations of $\delta_{\mathrm{HA}}$, but since the latter has less theoretical foundation $20.7 \mathrm{~J}^{1 / 2} \mathrm{~cm}^{-3 / 2}$ is to be preferred.

For a compound containing a $\mathrm{P}(\mathrm{S}) \mathrm{SH}$ group, the polar part of the cohesion energy is, of course, less than for the corresponding oxygenated compound. This is also reflected in the absence of any tendency to dimerization through hydrogen bonding compared with, for example, dibutylphosphate. The relatively small polarity is compensated by a higher dispersion part of cohesion energy due to the high polarizability of the sulfur atoms. It may, therefore, be assumed that the chosen set of nonpolar solvents has given a more accurate estimation of the solubility parameter than if some polar solvents capable of strong inductive effects had been considered. Induction forces generated by $\mathrm{HA}$ in a non-polar surrounding are expected to be of the same magnitude as for HA itself and they should, therefore, not have any influence on the observed $\delta_{\mathrm{HA}}$ in this case.

Acknowledgement. The author is indebted to Professor Jan Rydberg and Docent Jan-Olov Liljenzin for many helpful discussions.

\section{REFERENCES}

1. Wingefors, S. Acta Chem. Scand. A 34 (1980) 289.

2. Dyrssen, D. Sven. Kem. Tidskr. 64 (1952) 213.

3. Omori, T., Wakahayashi, T., Oki, S. and Suzuki, N. J. Inorg. Nucl. Chem. 26 (1964) 2265.

4. Wakahayashi, T., Oki, S., Omori, T. and Suzuki, N. J. Inorg. Nucl. Chem. 26 (1964) 2255.

5. Skytte-Jensen, B. In Dyrssen, D., Liljenzin, J.-O. and Rydberg, J., Eds., Solvent Extraction Chemistry, North-Holland, Amsterdam 1967, p. 517.

6. Irving, H. M. N. H. Ion Exchange and Solvent Extraction 6, Dekker, New York 1974, p. 139.

7. Hildebrand, J. H. and Scott, R. L. The Solubility of Non-Electrolytes, 3rd Ed., Dover, N.Y. 1964.

8. Hildebrand, J. H., Scott, R. L. and Prausnitz, J. M. Regular and Related Solutions, Van Nostrand-Reinhold, New York 1970.

9. Prausnitz, J. M. Molecular Thermodynamics of Fluid-Phase Equilibria, Prentice-Hall, Englewood Cliffs, N. J. 1969.

10. Weimer, R. F. and Prausnitz, J. M. Hydrocarbon Proc. 44 (1965) 237.

11. Siekierski, S. J. Inorg. Nucl. Chem. 24 (1962) 205.

12. Rozen, A. M. In Dyrssen, D., Liljenzin, J.-O. and Rydberg, J., Eds., Solvent Extraction Chemistry, North-Holland, Amsterdam 1967, p. 195.

13. Ref. 8 , pp. 75,183 .

14. Funk, E. W. and Prausnitz, J. M. Ind. Eng. Chem. 62 (1970) 8.

15. Hoy, K. L. J. Paint Technol. 42 (1970) 76.

16. Morawetz, E. J. Chem. Thermodyn. 4 (1972) 145.

17. Kabachnik, M. I., Mastrukova, T. A., Shipov, A. E. and Melentyeva, T. A. Tetrahedron 9 (1960) 10. 